Abstract FRI0555 - Table 1. Mean SWV and differences in the examined muscles between myositis patients and controls

\begin{tabular}{|c|c|c|c|c|c|}
\hline \multirow[t]{2}{*}{ Muscle } & \multicolumn{2}{|c|}{$\begin{array}{c}\text { Shear wave } \\
\text { velocity }(\mathrm{m} / \mathrm{s})\end{array}$} & \multirow{2}{*}{$\begin{array}{c}\text { Mean } \\
\text { difference } \\
(95 \% \mathrm{Cl})\end{array}$} & \multirow[t]{2}{*}{$\begin{array}{l}\text { Difference } \\
\text { percentage }\end{array}$} & \multirow[t]{2}{*}{$\begin{array}{c}\text { Significance } \\
\text { ( } p \text { value) }\end{array}$} \\
\hline & Healthy & Myositis & & & \\
\hline Vastus lateralis & 1.74 & 1.42 & $\begin{array}{c}-0.32(0.19 \\
0.46)\end{array}$ & $18.4 \%$ & $<0.001^{\star}$ \\
\hline Rectus femoris & 1.77 & 1.53 & $\begin{array}{c}-0.24(0.12, \\
0.34)\end{array}$ & $13.6 \%$ & $<0.001^{*}$ \\
\hline Vastus medialis & 1.66 & 1.45 & $\begin{array}{c}-0.21(0.06 \\
0.35)\end{array}$ & $12.7 \%$ & $<0.001^{*}$ \\
\hline Vastus intermedius & 1.89 & 1.60 & $\begin{array}{c}-0.29(0.11 \\
0.47)\end{array}$ & $15.3 \%$ & $<0.001^{*}$ \\
\hline Biceps brachii & 1.94 & 1.79 & $\begin{array}{c}-0.15(-0.01 \\
0.30)\end{array}$ & $7.7 \%$ & 0.088 \\
\hline Biceps femoris & 1.73 & 1.38 & $\begin{array}{c}-0.35(0.24 \\
0.46)\end{array}$ & $20.2 \%$ & $<0.001^{*}$ \\
\hline Semitendinosus & 1.72 & 1.45 & $\begin{array}{c}-0.27(0.09 \\
0.43)\end{array}$ & $15.7 \%$ & $<0.001^{*}$ \\
\hline Semimembranosus & 1.75 & 1.38 & $\begin{array}{c}-0.37(0.28, \\
0.45)\end{array}$ & $21.1 \%$ & $<0.001^{*}$ \\
\hline
\end{tabular}

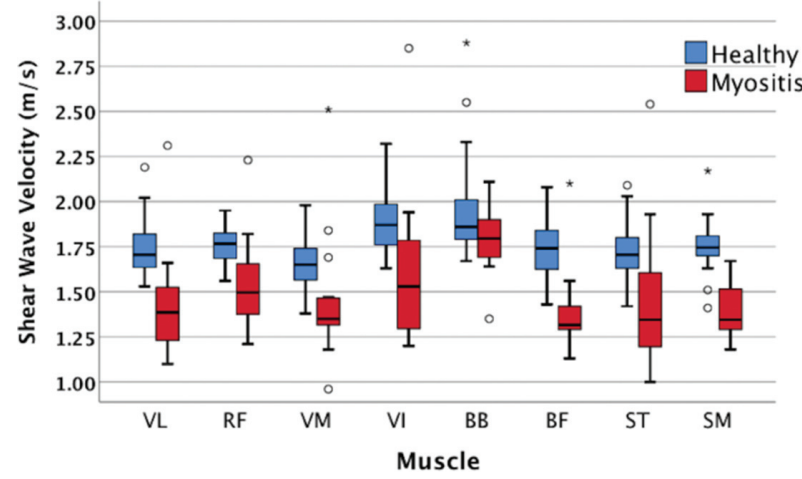

Conclusions: Muscle elasticity in myositis patients has been shown to be $21 \%$ lower in comparison to healthy participants and seems to moderately correlate with disease activity, muscle strength and function. To our knowledge, this is the first study to show that shear wave elastography can detect changes in muscle elasticity in myositis patients. Further validation is required to evaluate the value of this novel ultrasound technology as an imaging biomarker for myositis.

Disclosure of Interest: None declared

DOI: 10.1136/annrheumdis-2018-eular.1928

\section{FRI0556 AUTOMATED SCORING OF KNEE OSTEOARTHRITIS (OA) ON ROUTINE RADIOGRAPHS IDENTIFIES DISEASE SEVERITY IN OA}

A. Law ${ }^{1}$, L. Assi ${ }^{1}$, A. Harrison ${ }^{1}$, F.A. Howe ${ }^{2}$, N. Sofat ${ }^{1} .{ }^{1}$ Institute of Infection and Immunity; ${ }^{2}$ Molecular and Clinical Sciences Research Institute, St George's, University of London, London, UK

Background: Knee osteoarthritis $(\mathrm{OA})$ causes pain and limited function of the knee, linked to cartilage loss, bone remodelling and inflammation ${ }^{1}$.

Objectives: We aimed to find out whether joint space measures can stratify patients into mild and advanced OA using an automated scoring tool ${ }^{2}$.

Methods: 86 weight-bearing radiographs (target joint: 41 left, 45 right knee) were analysed by using ImageBiopsy Lab software (JSx). Automated joint space (JS) measures were joint space width (JSW), minimum height $(\mathrm{minH})$ and joint space area/region of interest (JSA/ROI, in $\mathrm{mm}^{2}$ ). JS measures were compared against Western Ontario and McMaster Universities Osteoarthritis Index (WOMAC) of pain, stiffness and function, with each subscale score normalised to a total score of 100 . Body-mass index (BMI) and age data were available as potential covariates. Image analysis was initiated with landmarks automatically placed on the end of tibial plateau for each compartment, with fine manual adjustment of landmarks and contour spline if necessary. Clinical scores were compared between mild OA $(n=24)$ and advanced OA $(n=62)$; patient groups were defined according to their clinical pathway of standard care: medical management for early OA and knee replacement for advanced OA. SPSS Statistics 25 was used for analysis. An independent-samples t-test was used to compare the automated radiological and clinical scores between the groups of different OA severity. Subsequently the General Linear Model (GLM) was used to evaluate group differences with appropriate covariates and fixed factors.
Results: Joint space measures were strongly linked to the two clinical groups of $\mathrm{OA}$ severity (mild and advanced $\mathrm{OA}$ ). Differences were significant in the medial JSA/ROI $(p<0.001)$, medial JSW $(p=0.001)$, medial MinH $(p<0.001)$, WOMAC pain $(p=0.001)$, stiffness and function (both $p=0.003)$. Age $(p=0.014)$ and $B M$ $(p=0.019)$ were also different between the two groups of OA severity in the t-test and were then used as co-variates in the GLM analysis along with gender and ethnicity as fixed factors. Medial JSA/ROI $(p=0.027)$ and medial MinH $(p=0.041)$ were still significantly different between mild and advanced $\mathrm{OA}$ in the multivariate test. Receiving Operating Characteristic (ROC) curves showed that medial JSA $\mathrm{ROI}$ was the better discriminator for severity of $\mathrm{OA}(\mathrm{AUC}=0.738)$ when compared to WOMAC scores ( $\mathrm{AUC}=0.719$ for WOMAC pain).

A
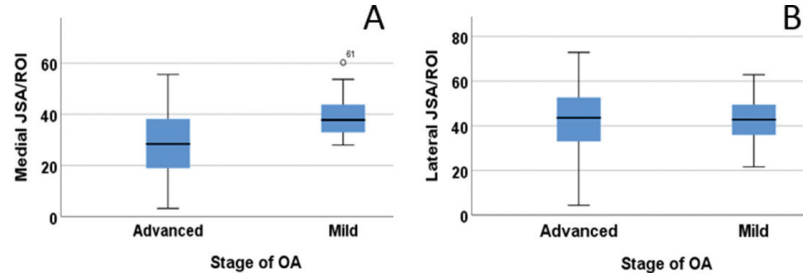

B

Abstract FRI0556 - Figure 1. Boxplots showing the relationship between stage of OA (mild and advanced) and (A) medial JSA/ROI, (B) lateral JSA/ROI

Conclusions: Medial joint space measures are possible markers for identifying the stage of disease if only radiographs were used. In particular, medial JSA/ROI may be utilised as an automated tool for characterising patient severity.

\section{REFERENCES :}

[1] Kuttapitiya A, Assi L, Laing K, et al. Microarray analysis of bone marrow lesions in osteoarthritis demonstrates upregulation of genes implicated in osteochondral turnover, neurogenesis and inflammation. Annals of the Rheumatic Diseases 2017;76(10):1764-1773.

[2] Hunter D, Altman R, Cicuttini F, et al. OARSI Clinical Trials Recommendations: Knee imaging in clinical trials in osteoarthritis. Osteoarthritis and Cartilage 2015;23(5):698-715.

Acknowledgements: The software for analysis in this study was provided by ImageBiopsy Lab and all analyses were performed independently at St George's, University of London.

Disclosure of Interest: None declared

DOI: 10.1136/annrheumdis-2018-eular.5304

\section{FRI0557 THERMOGRAPHIC ANALYSIS OF HANDS AND WRISTS OF RHEUMATOID ARTHRITIS PATIENTS}

A. Gatt ${ }^{1}$, A. Borg ${ }^{2}$, C. Mercieca ${ }^{2}$, A. Grech Scalpello ${ }^{1}$, L. Camilleri ${ }^{3}$, C. Gatt ${ }^{2}$, N. Chockalingam ${ }^{4}$, C. Formosa ${ }^{1} .{ }^{1}$ Faculty of Health Sciences; ${ }^{2}$ Faculty of Medicine and Surgery; ${ }^{3}$ Faculty of Science, University of Malta, Msida, Malta; ${ }^{4}$ Faculty of Health, Staffordshire University, Stoke on Trent, UK

Background: Thermography has been utilised in a number of studies in rheumatoid arthritis (RA), ${ }^{1}$ however there is a paucity of evidence as regards to the possibility of applying this non-invasive technology to the detection of synovitis of the hands and wrists. With normative data having been already published, ${ }^{2}$ it is now possible to compare the thermographic characteristics of RA patients without active synovitis to those with a normal thermal distribution in order to determine the baseline characteristics of RA hands and wrists. This would consequently provide a foundation against which further studies investigating RA patients with synovitis can be compared.

Objectives: To determine whether rheumatoid arthritis (RA) patients without active synovitis in their hands exhibit different baseline thermographic patterns of the fingers and palms when compared to healthy individuals

Methods: Data from 31 RA patients were compared to 51 healthy controls. Inclusion criteria were confirmed absence of synovitis by clinical examination and musculoskeletal ultrasound in rheumatoid patients. Thermographic imaging of the regions of interest (ROIs) were obtained as per established protocols ${ }^{2}$

Results: Significant differences were found between the mean temperatures of the palm regions and fingers of the healthy participants when compared to the palm regions and fingers of their RA counterparts $(p=0.001)$, with the latter group exhibiting higher temperatures in all ROls. No significant differences were found between ROls of the palms and fingers of both hands in either group. Logistic regression models confirm that both palm and finger temperature increase significantly in RA 